\title{
The Role of Omega-3 Polyunsaturated Fatty Acids in Stroke
}

\author{
Jiyuan Bu, Yang Dou, Xiaodi Tian, Zhong Wang, and Gang Chen \\ Department of Neurosurgery \& Brain and Nerve Research Laboratory, The First Affiliated Hospital of Soochow University, \\ 188 Shizi Street, Suzhou 215006, China \\ Correspondence should be addressed to Gang Chen; nju_neurosurgery@163.com
}

Received 29 January 2016; Revised 16 May 2016; Accepted 26 May 2016

Academic Editor: Qian Liu

Copyright @ 2016 Jiyuan Bu et al. This is an open access article distributed under the Creative Commons Attribution License, which permits unrestricted use, distribution, and reproduction in any medium, provided the original work is properly cited.

\begin{abstract}
Stroke is the third commonest cause of death following cardiovascular diseases and cancer. In particular, in recent years, the morbidity and mortality of stroke keep remarkable growing. However, stroke still captures people attention far less than cardiovascular diseases and cancer. Past studies have shown that oxidative stress and inflammation play crucial roles in the progress of cerebral injury induced by stroke. Evidence is accumulating that the dietary supplementation of fish oil exhibits beneficial effects on several diseases, such as cardiovascular diseases, metabolic diseases, and cancer. Omega-3 polyunsaturated fatty acids (n-3 PUFAs), the major component of fish oil, have been found against oxidative stress and inflammation in cardiovascular diseases. And the potential of n-3 PUFAs in stroke treatment is attracting more and more attention. In this review, we will review the effects of n-3 PUFAs on stroke and mainly focus on the antioxidant and anti-inflammatory effects of n-3 PUFAs.
\end{abstract}

\section{Introduction}

Stroke, also known as cerebrovascular insult or brain attack, is defined by World Health Organization as "neurological deficit of cerebrovascular cause that persists beyond 24 hours or is interrupted by death within 24 hours" in the 1970s [1]. Stroke was firstly reported in the 2nd millennium BC, and firstly described by Hippocrates. But up till now, the systematic treatment strategy of stroke remains elusive.

In 1946, Hansen and Burr found that the Eskimos who live in Newfoundland rarely suffer from cardiovascular disease [2]. They owe the beneficial effects to the diet of Eskimos, which is rich in fish and seafood. Fish oil begins to capture people's attention. Further studies indicated that the benefit effects of fish oil are mainly mediated by omega- 3 polyunsaturated fatty acids (n-3 PUFAs), which are against a range of diseases, including cardiovascular diseases, inflammatory diseases like arthritis, metabolic diseases like type 2 diabetes, and cancer [3].

The aim of this paper is to summarize the research progress of n-3 PUFAs, especially the effects on stroke.

\section{Subsets, Sources, and Metabolism of n-3 PUFAs}

According to the number of double bonds in fatty acid side chains, the natural fats are classified into 3 subsets: saturated, monounsaturated, and polyunsaturated. The classification of fatty acids is shown in Figure 1. And there is a fourth artificial subset, trans fats, which is created by hydrogenation [4]. Polyunsaturated fats are further classified into 2 subsets by the first double bond: omega- 3 fatty acids and omega6 fatty acids. n-3 PUFAs have the first double bond at the third carbon from the methyl terminal, whereas omega- 6 polyunsaturated fatty acids (n-6 PUFAs) have the first double bond at the sixth carbon [5]. Mammalian cells are short of the desaturase that can convert n-6 to n-3 PUFAs, which means that n-3 PUFAs must be supplied with the diet. Fish, such as mackerel, salmon, sardines, halibut, herring, and tuna, in the human diet is the major source of n-3 PUFAs, containing docosahexaenoic acid (DHA) and eicosapentaenoic acid (EPA). Quite a few kinds of vegetables and vegetable oil, such as flaxseeds, canola, pumpkin seeds, flaxseed oil, canola oil, and perilla seed oil, also can provide n-3 PUFAs, such as 


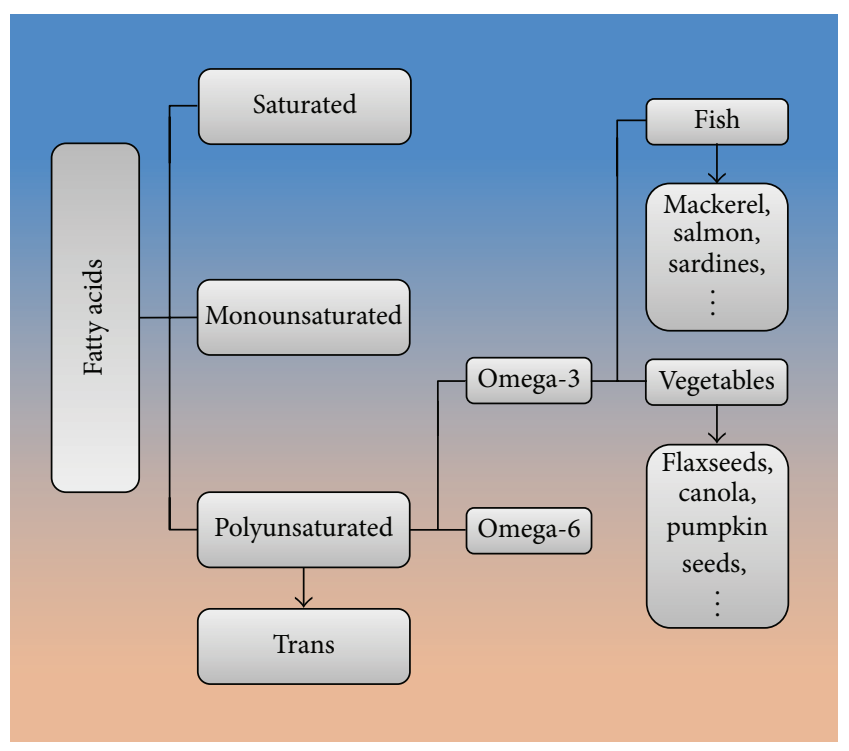

FIgURE 1: The classification of fatty acids.

alpha-linolenic acid (ALA), which can be converted to EPA and further to DHA by a desaturase enzyme [6]. Isotopelabeled ALA trials suggested that the conversion of natural ALA to EPA is between $0.2 \%$ and $21 \%$ and further to DHA is between $0 \%$ and $9 \%$ [7]. The conversion of ALA to DHA and EPA is likely influenced by the competitive inhibition of linoleic acid and negative feedback of DHA and EPA [8]. And the interconversion is limited. So the best way to increase fatty acids intake is to supplement them with specific fatty acids [9].

\section{Research Tools for n-3 PUFAs}

3.1. Gas Chromatography Methods. All fatty acids in plasma fraction can be analyzed by high performance liquid chromatography and mass spectrometry.

3.2. Fat-1 Transgenic Mice. Mammalian cells are short of the desaturase, which can convert n-6 to n-3 PUFAs [10]. Fat-1 transgenic mice carrying a fat-1 gene expressed a Caenorhabditis elegans desaturase that introduces a double bond into n-6 PUFAs to form n-3 PUFAs [11]. Due to the capability, fat-1 transgenic mice are able to produce n-3 PUFAs from n-6 PUFAs and their organs or tissues are rich in n-3 PUFAs without the dietary n-3 PUFAs supplementation [12]. Accordingly, fat-1 transgenic mice can avoid the potential confounding effects from the dietary supplementation [13]. The fat-1 transgenic mice are widely used as new tools for $n-3$ PUFAs studies.

3.3. Administration Pathway. Rats are chosen in most experimental studies, and some studies also use mice, baboons, and piglets as animal models [14]. The common administration way is oral administration or intragastric administration. The dosage of oral drugs is $0.2 \mathrm{~g}$ to $30 \mathrm{~g}$ of EPA and
DHA/kilogram, and the duration of intervention for studies is from $24 \mathrm{~h}$ to 4 weeks [15].

\section{4. n-3 PUFAs and Stroke}

4.1. Stroke. Stroke has two main types: ischemic stroke, due to the lack of blood flow, and hemorrhagic stroke, due to the bleeding. Ischemic stroke can be further classified into cerebral infarction and transient ischemic attack (TIA), and hemorrhagic stroke also can be further classified into subarachnoid hemorrhage (SAH) and intracerebral hemorrhage (ICH).

4.2. n-3 PUFAs and Ischemic Stroke. Cerebral infarction is defined as the necrosis of the cerebral tissue caused by ischemia. Under normal circumstances, cerebral blood flow $(\mathrm{CBF})$ is $50 \pm 10 \mathrm{~mL} / 100 \mathrm{~g} / \mathrm{min}$. When $\mathrm{CBF}$ drops to $15 \mathrm{~mL} / 100 \mathrm{~g} / \mathrm{min}$, cerebral cortical evoked potential and brain waves disappear completely, but cerebral cells are still alive. And when CBF drops to $8-10 \mathrm{~mL} / 100 \mathrm{~g} / \mathrm{min}$, even lower, the function of ion pumps in neuron membrane begins to fail, inducing potassium efflux and sodium influx, and cerebral cells begin to die and cerebral infarction occurs. Traditionally, TIA was defined as the episodes of neurologic dysfunction resulting from focal cerebral ischemia and completely recovers within 24 hours [16]. The American Heart Association renewed the definition in 2009 and changed the definition from time-based to tissue-based [15]. The newest diagnosis of TIA is based on the restricted diffusion on MRI [16]. Currently, the diagnosis of TIA is dependent upon CT or MRI findings heavily. Cerebral ischemia/reperfusion (I/R) injury is a phenomenon that ischemic stroke induces cerebral cells damage, and, after the restoration of hemoperfusion, the ischemic injury even becomes more serious.

Early reperfusion is desirable, but reperfusion also induces additional neural tissue injury and the breakdown of cellular integrity by oxidative stress, excitotoxic signaling, inflammation, and others [17]. Elevated oxidative stress is associated with the pathogenesis of cerebral injury in I/R [18]. During cerebral I/R, the endogenous antioxidative defense systems turn to be ineffective, which results from the inactivation of detoxification systems and the degradation of antioxidants $[19,20]$. A multitude of oxygen radicals such as reactive oxygen species (ROS) begin to accumulate and cause apoptosis and cellular damage [21]. ROS are involved in the oxidative damage of proteins, nucleic acids, and lipids in ischemic tissues directly [22]. ROS can also cause lipid peroxidation, which leads to the damage of biological membranes [23]. Classic description of lipid peroxidation mainly contains three steps [24]. First, a hydrogen atom removes from the side chain of polyunsaturated fatty acids, forming the lipid radical. Then the unpaired electron rearranges, forming conjugated dienes. And the lipid radical converts into lipid peroxyl radical by attracting molecular oxygen. Second, the lipid peroxyl radical extracts a hydrogen atom and begins a cycle of peroxidation reaction. Third, two radicals combine and form a nonradical. Beside hydroperoxides, lipid peroxidation also produces aldehydes, lipid hydroxides, and others. The lipid 


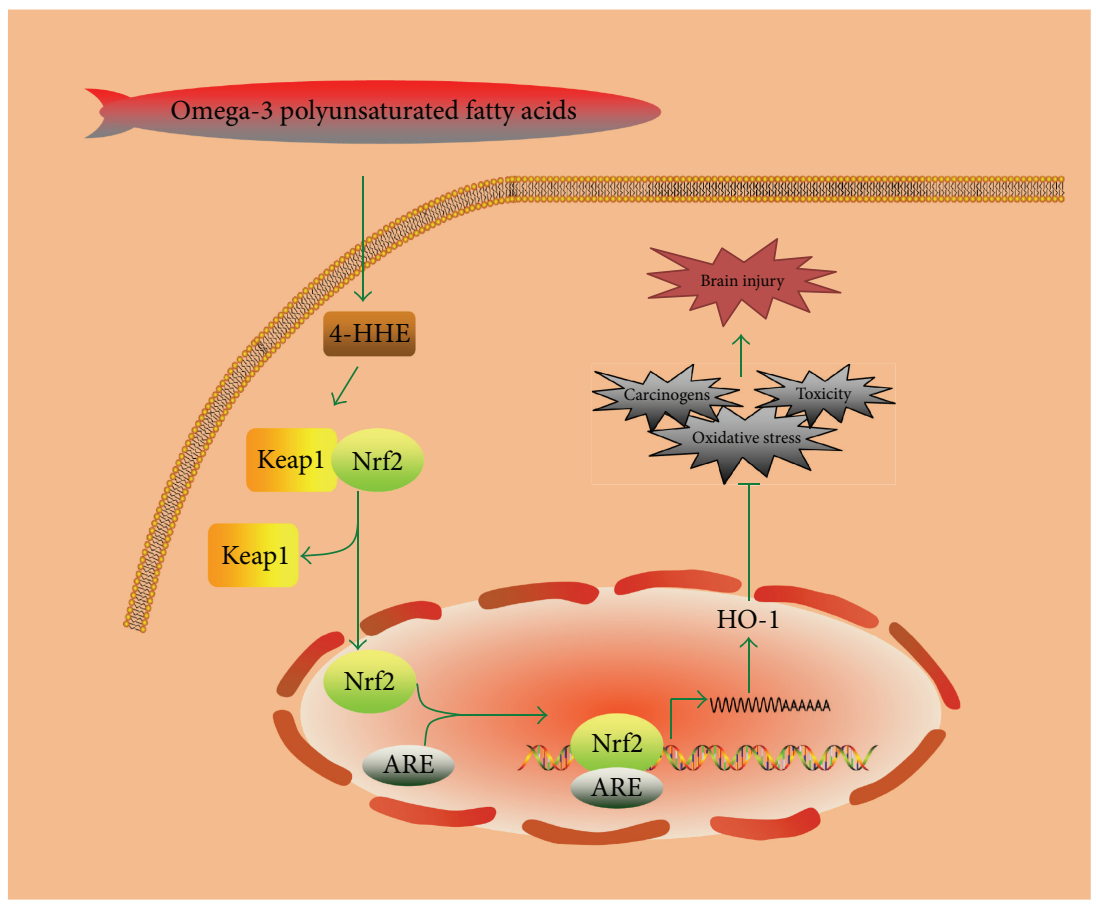

Figure 2: The Nrf2/HO-1 signaling pathway induced by n-3 PUFAs.

peroxidation of n-3 PUFAs is a complex process and ultimately produces the 4-hydroxynonenal. The peroxidation of n-3 PUFAs majorly produces the 4-hydroxy-2E-hexenal (4-HHE); the 4-hydroxy-2E-nonenal (4-HNE) is the major product of n-6 PUFAs, and some other fatty acids produce 4-hydroxy-2E, 6Z-dodecadienal (4-HDDE) [25]. The lipid peroxidation will accumulate these products and affect the normal cell functions, leading to cell death at last. The peroxidation of n-3 PUFAs belongs to nonenzymatic lipid peroxidation, which derived from free radical reactions [26]. The reaction between ROS and transition metals produce the hydroxyl radical, the major radical in this process. The lipid peroxidation will indicate the overproduction of ROS, and this vicious circle may cause the increase of ROS, necrosis, and apoptosis during the time [27]. In addition, the generation of excessive ROS reduces the activation and bioavailability of NO [28]. Oxidative stress and ROS are detrimental factors in the progression of cerebral I/R injury [29]. Oxidative stress can increase the expression of cytokine and the occurrence of edema and apoptosis [30]. During reperfusion, ROS acts as the signaling molecules, inducing the activation of NF- $\kappa \mathrm{B}$ and activator protein-1 (AP-1) [31]. Due to the low activities of antioxidant enzymes and the high rates of oxidative metabolic activities, neurons in the brain are more vulnerable to ischemic damage [32]. Free radical generation, calcium overload, excitatory neurotransmitter accumulation, inflammation, and apoptosis are all related to neuronal injuries after ischemic damage $[33,34]$.

The dietary supplementation of n-3 PUFAs can decrease the volume of cerebral infarction partly by adjusting antioxidant enzymes activities and partly by working as an antioxidant directly [35]. n-3 PUFAs may act as an antioxidant in reducing cerebral lipid peroxides and play a role in regulating oxidative stress through the increase of oxidative burden and the improvement of antioxidative defense capacity [36]. The chronic administration and dietary supplementation of $n-3$ PUFAs can improve symptoms of cerebral I/R by increasing the antioxidative capacity, as well as reducing the induction of chaperon molecules and the stabilization of membrane integrity and lipid peroxidation [37]. The dietary supplementation of ALA is also found such that it can reduce the level of lipid peroxidation, as well as increasing the risk of spontaneous reperfusion [38]. The neuroprotective effects of n-3 PUFAs include not only inhibiting the oxidative stress but also enhancing the expression of nuclear factor E2-related factor 2 (Nrf2)/heme oxygenase-1 (HO-1) [39]. The Nrf2/HO-1 signaling pathway induced by $n-3$ PUFAs is shown in Figure 2. Nrf2/HO-1 signaling pathway is a crucial mechanism of n-3 PUFAs for protecting cells [40]. n-3 PUFAs reduce ischemic injury by activating $\mathrm{Nrf} 2$ and increasing $\mathrm{HO}-$ 1 production [41]. The protective mechanisms are associated with the upregulation of $\mathrm{HO}-1$, the activation of $\mathrm{Nrf} 2$, and the oxidation of 4-HHE. 4-HHE is the end-product of n-3 PUFAs by peroxidation and acts as an effective Nrf2 inducer [42]. Nrf2 acts as a transcription factor in regulating phase2 enzymes expression. Under normal conditions, Kelch-like ECH-associated protein-1 (Keap1), the inhibitory protein of $\mathrm{Nrf2}$, will bind to Nrf2 and lead Nrf2 to the proteasomal degradation process. Under oxidative stress, 4-HHE will react with the cysteine residues of Keapl and dissociate Nrf2 from Keap1 [41]. Then Nrf2 will translocate into the nucleus, bind to antioxidant responsive element (ARE), and induce the expression of phase-2 enzymes [43]. Phase2 enzymes like HO-1 mainly mediate the cytoprotection 
against oxidative stress, carcinogens, and toxicity. Cerebral ischemia/reperfusion injury will cause the elevated expression of antioxidative proteins and Keap1/Nrf2 system and increase the expression of Nrf2 and HO-1, which can inhibit the activation of microglia and the expression of proinflammatory cytokine [44]. The shortage of Nrf2 or HO-1 will sensitize animals to inflammation and injury induced by ischemic stroke. The neuroprotection against cerebral ischemic stroke mediated by DHA includes improving neuronal defense capacity and inhibiting cellular inflammatory mechanisms by increasing the expression of Nrf2 and HO-1 [45]. Although DHA itself is able to increase the expression of Nrf2 and HO-1 in glial cell cultures, it is not enough to induce the promotion of Nrf2 and HO-1 in vivo. Actually, the treatment with DHA after ischemic stroke only can provide a driving force for the promotion of Nrf2 and HO-1 [46].

On the other side, n-3 PUFAs exhibit the modulatory effects on the homeostasis of redox potential by enhancing the oxidative burden induced by lipid peroxidation and activating the activity of antioxidant enzymes [47]. Under normal conditions, n-3 PUFAs such as DHA may not act as initiators of free radical generation, while in the oxidized environments DHA may augment the oxidative burden. That is to say, n-3 PUFAs treatments may lead to augmenting, or be comparable at least to, the damage induced by cerebral I/R injury [48]. People found that DHA could increase the activity of MPO, the expression of COX-2 mRNA, and the activity of caspase 3 , which will exacerbate neurobehavioral deficits and cerebral infarction in the end [49]. The acute posttreatments with DHA after cerebral ischemic stroke are found to augment oxidative burden and subsequently exacerbate cerebral I/R injury remarkably [37]. The detrimental effects of DHA in cerebral I/R are associated with oxidative changes. DHA alone has little effects on the generation of free radical in neuroglia but increases the oxidative burden induced by hydrogen peroxide greatly. The high level of free n-3 PUFAs will induce free radicals to react with unsaturated fatty acids instantaneously [37]. Once beginning, the reaction will continue and propagate an amplification cycle of free radicals generation, which will lead to the augmented oxidative stress and increase the oxidative burden induced by cerebral I/R significantly.

In terms of inflammation, ischemic stroke will trigger complex cellular responses, including the recruitment of inflammatory cells and the activation of glial cells [50]. Leukocytes will move in the interstitial compartments and release the proteolytic enzymes and cytotoxic metabolites, inducing the nerve cells death and enhancing the deleterious effects of ischemic stroke. In the end, leukocytes plugging in capillaries, the aggregation of platelet leukocyte, and the extravasation of albumin occur [51]. n-3 PUFAs are found to inhibit systemic inflammatory responses and modulate vascular inflammation by changing intracellular signal transduction and controlling lipid mediators [52]. The anti-inflammatory effects of n-3 PUFAs include inhibiting the conversion of arachidonate acids to the proinflammatory lipid intermediates, interrupting the NF- $\kappa \mathrm{B}$ signaling pathway, and activating the AMP-activated protein kinase, inducing the synthesis of anti-inflammatory lipid mediators like resolvins and protectins [40]. DHA is the precursor of neuroprotectin D1 (NPD1) and NPD1 can downregulate apoptosis, promote neurogenesis, and inhibit leukocyte infiltration and the expression of proinflammatory gene [53]. n3 PUFAs also exhibit potent immunomodulatory effects by reducing the leukocyte chemotaxis and inhibiting the expression of adhesion molecules [54]. EPA and DHA can exhibit neuroprotective effects through inducing the expression of receptors of chemoattractants and inhibiting the activation of macrophages and microglia and the migration of neutrophils and monocytes. DHA also can increase the generation of antiapoptotic proteins such as $\mathrm{Bcl}-\mathrm{xL}$ and $\mathrm{Bcl}-2$, which inhibit the inflammatory response mediated by microglial cells [55]. In glial cell culture, DHA exhibits immunosuppressive effects by reducing the phosphorylation of c-Jun N-terminal kinase (JNK) and c-Jun and inhibiting the activation of AP-1 [56]. The activation of JNK plays a crucial role in neuroinflammation and cell death induced by ischemic stroke [57]. Once activated, JNK will increase the phosphorylation of c-Jun, the crucial component of AP-1, and induce the cell death program and transcription-dependent inflammation [58]. The downregulation of JNK/AP-1 signaling pathway, which includes decreasing the phosphorylation of c-Jun and JNK and inhibiting the DNA-binding activity of AP1 , contributes to the neuroprotective effects of DHA against cerebral ischemic stroke [59]. Someone found that $G$ proteincoupled receptor 120 (GPR120) could be activated by longchain fatty acids and GPR120 acted as a functional receptor or sensor of n-3 PUFAs, exerting the anti-inflammatory effects [60]. Through GPR120, n-3 PUFAs inhibit the activation and phosphorylation of TAK1 by the $\beta$-arrestin $2 / \mathrm{TAB} 1$ dependent effect, resulting in the inhibition of TNF-a and TLR inflammatory signaling pathways [61].

4.3. $n$-3 PUFAs and Hemorrhagic Stroke. SAH is a pathologic syndrome defined by the appearance of the blood in the subarachnoid space resulting from a wide variety of causes. The most common cause of SAH is trauma, and $85 \%$ of nontraumatic patients are in case of underlying cerebral aneurysm $[62,63]$; the other $15 \%$ are idiopathic [64]. Two-thirds of the idiopathic patients are due to perimesencephalic hemorrhage $[65,66]$. According to unenhanced CT, SAH is classified into mainly three distinct forms [67]. Aneurysm rupture and vascular malformation belong to the first form, in which $\mathrm{SAH}$ is centered in the central basal or suprasellar cisterns and extends to periphery diffusely [68]. Idiopathic perimesencephalic hemorrhage resulting from aneurysm rupture, vascular malformation, and cervicomedullary junction tumor belong to the second form, in which SAH is centered in the low basal or perimesencephalic cisterns and does not extend. The third form, in which SAH is centered in the cerebral convexities, includes cerebral amyloid angiopathy, reversible cerebral vasoconstriction syndrome, cerebral venous thrombosis, and posterior reversible encephalopathy syndrome. There are several reasons behind the morbidity and mortality of the patients with SAH and cerebral vasospasm (CV) is a significant one of them [69]. The pathogenesis of cerebral vasospasm is still unclear. Inflammation, Endothelin (ET), $\mathrm{NO}$, and products of erythrocyte degradation all have been 
confirmed to play crucial roles in $\mathrm{CV}[70,71]$. OxyHb, produced by erythrocyte degradation, is one of the causes of CV. When blood flows into the subarachnoid space and soak vessels for a long time, dysfunction of vessels occurs and then the blood cells begin to collapse and lipid peroxide and free radicals produced [72]. The products lead to a series of chain reactions, the destruction of biological membrane, the removal of endogenous $\mathrm{NO}$, and the increasing production of ET. The diastolic and systolic function of vessels failed at last, leading to cerebral hemorrhage [73]. Quite a few studies are indicative of the fact that Rho-kinase plays a crucial role in CV [74, 75], and some agents like thromboxane A2 (TXA2) and sphingosylphosphorylcholine (SPC) can activate Rho-kinase [76, 77]. Recently, EPA is reported to inhibit SPC by inducing the activation of Rho-kinase in vitro [78]. Moreover, EPA can change the concentration of arachidonic acid, which has a potential role in CV [79] and inhibit the synthesis of TXA2 [80]. The concentration of free fatty acids increases after SAH and has a secondary elevation between 8 and 10 days after SAH [79]. These observations are suggestive of the fact that EPA can inhibit CV after SAH and improve clinical prognosis by inhibiting the activation of Rho-kinase [81]. Furthermore, oral EPA is found to reduce the risk of CV after SAH [82], and using n-3 PUFAs for immunomodulatory interventions could reduce the risk of delayed cerebral ischemia after SAH [83].

$\mathrm{ICH}$ is a pathologic syndrome caused by the rupture of intracranial vessel, which is resulting from nontraumatic factors, and the appearance of the blood in the intracerebral space leads to several causes. Because n-3 PUFAs demonstrate poor effects on $\mathrm{ICH}$, there are a few studies focusing on n3 PUFAs and ICH. A slice of studies suggests that different concentration of n-3 PUFAs leads to different effects [84]. Low levels of n-3 PUFAs protect against thrombogenesis, while high levels may induce oxidative damage and become a risk factor for ICH [85]. Moreover, high levels may lead to a poor functional outcome and a severe motor impairment after ICH [86].

\section{Expectation}

Except the antioxidant and anti-inflammatory effects, n-3 PUFAs also can trigger other responses like neuranagenesis and revascularization in stroke. The classification of fatty acids is shown in Figure 1, and the $\mathrm{Nrf} 2 / \mathrm{HO}-1$ signaling pathway induced by n-3 PUFAs is shown in Figure 2. Even though n-3 PUFAs is generally accepted as a beneficial factor in diets, there are still many debates remaining. As most of the previous studies focus on the prevention and post-treatments of stroke, the lack of systematic treatment strategy with n-3 PUFAs remained to be supplemented.

\section{Abbreviations}

n-3 PUFA: Omega-3 polyunsaturated fatty acids

N-6 PUFA: Omega-6 polyunsaturated fatty acids

DHA: Docosahexaenoic acid

EPA: $\quad$ Eicosapentaenoic acid

ALA: Alpha-linolenic acid
TIA: Transient ischemic attack

SAH: Subarachnoid hemorrhage

ICH: Intracerebral hemorrhage

CBF: Cerebral blood flow

I/R: Ischemia/reperfusion

ROS: $\quad$ Reactive oxygen species

4-HHE: 4-Hydroxy-2E-hexenal

4-HNE: 4-Hydroxy-2E-nonenal

4-HDDE: 4-Hydroxy-2E, 6Z-dodecadienal

AP-1: Activator protein-1

Nrf2: $\quad$ Nuclear factor E2-related factor

HO-1: Heme oxygenase-1

Keap1: Kelch-like ECH-associated protein-1

ARE: Antioxidant responsive element

NPD1: Neuroprotectin D1

JNK: c-Jun N-terminal kinase

GPR120: G protein-coupled receptor 120

CV: $\quad$ Cerebral vasospasm

ET: $\quad$ Endothelin

TXA2: Thromboxane A2

SPC: $\quad$ Sphingosylphosphorylcholine.

\section{Competing Interests}

The authors declare that they have no competing interests.

\section{Authors' Contributions}

Jiyuan Bu and Yang Dou contributed equally to this work.

\section{References}

[1] World Health Organization, Cerebrovascular Disorders, World Health Organization, Geneva, Switzerland, 1978.

[2] A. E. Hansen and G. O. Burr, "Essential fatty acids and human nutrition," Journal of the American Medical Association, vol. 132, no. 14, pp. 855-859, 1946.

[3] P. C. Calder, "Functional roles of fatty acids and their effects on human health," Journal of Parenteral and Enteral Nutrition, vol. 39, supplement 1, pp. 18S-32S, 2015.

[4] A. P. DeFilippis and L. S. Sperling, "Understanding omega-3's," American Heart Journal, vol. 151, no. 3, pp. 564-570, 2006.

[5] IUPAC-IUB Commission on Biochemical Nomenclature, "The nomenclature of lipids," Lipids, vol. 12, no. 6, pp. 455-468, 1977.

[6] C. Gomez-Candela, M. C. Roldan Puchalt, S. Palma Milla, B. Lopez Plaza, and L. Bermejo, "The role of omega-3 fatty acids in diets," Journal of the American College of Nutrition, vol. 34, supplement 1, pp. 42-47, 2015.

[7] G. Burdge, " $\alpha$-linolenic acid metabolism in men and women: nutritional and biological implications," Current Opinion in Clinical Nutrition and Metabolic Care, vol. 7, no. 2, pp. 137-144, 2004.

[8] P. L. L. Goyens, M. E. Spilker, P. L. Zock, M. B. Katan, and R. P. Mensink, "Conversion of $\alpha$-linolenic acid in humans is influenced by the absolute amounts of $\alpha$-linolenic acid and linoleic acid in the diet and not by their ratio," The American Journal of Clinical Nutrition, vol. 84, no. 1, pp. 44-53, 2006.

[9] L. M. Arterburn, E. B. Hall, and H. Oken, "Distribution, interconversion, and dose response of n-3 fatty acids in humans," 
The American Journal of Clinical Nutrition, vol. 83, no. 6, supplement, pp. 1467S-1476S, 2006.

[10] C. Gravaghi, K. M. D. La Perle, P. Ogrodwski et al., "Cox-2 expression, $\mathrm{PGE}_{2}$ and cytokines production are inhibited by endogenously synthesized $n-3$ PUFAs in inflamed colon of fat- 1 mice," The Journal of Nutritional Biochemistry, vol. 22, no. 4, pp. 360-365, 2011.

[11] J. X. Kang, "Fat-1 transgenic mice: a new model for omega-3 research," Prostaglandins Leukotrienes and Essential Fatty Acids, vol. 77, no. 5-6, pp. 263-267, 2007.

[12] S. Bilal, O. Haworth, L. Wu, K. H. Weylandt, B. D. Levy, and J. X. Kang, "Fat-1 transgenic mice with elevated omega-3 fatty acids are protected from allergic airway responses," Biochimica et Biophysica Acta (BBA)-Molecular Basis of Disease, vol. 1812, no. 9, pp. 1164-1169, 2011.

[13] J. Griffitts, D. Saunders, Y. A. Tesiram et al., "Non-mammalian fat-1 gene prevents neoplasia when introduced to a mouse hepatocarcinogenesis model. Omega-3 fatty acids prevent liver neoplasia," Biochimica et Biophysica Acta-Molecular and Cell Biology of Lipids, vol. 1801, no. 10, pp. 1133-1144, 2010.

[14] S. Ghasemifard, G. M. Turchini, and A. J. Sinclair, "Omega-3 long chain fatty acid 'bioavailability': a review of evidence and methodological considerations," Progress in Lipid Research, vol. 56, pp. 92-108, 2014.

[15] N. Kaur, V. Chugh, and A. K. Gupta, "Essential fatty acids as functional components of foods-a review," Journal of Food Science and Technology, vol. 51, no. 10, pp. 2289-2303, 2014.

[16] C. Mijalski and B. Silver, "TIA management: should TIA patients be admitted? Should TIA patients get combination antiplatelet therapy?" The Neurohospitalist, vol. 5, no. 3, pp. 151$160,2015$.

[17] J. Sun, Z. Ling, F. Wang et al., "Clostridium butyricum pretreatment attenuates cerebral ischemia/reperfusion injury in mice via anti-oxidation and anti-apoptosis," Neuroscience Letters, vol. 613, pp. 30-35, 2016.

[18] S. Zhang, Y. Zhang, H. Li et al., "Antioxidant and antiexcitotoxicity effect of Gualou Guizhi decoction on cerebral ischemia/reperfusion injury in rats," Experimental and Therapeutic Medicine, vol. 9, no. 6, pp. 2121-2126, 2015.

[19] P. H. Chan, "Role of oxidants in ischemic brain damage," Stroke, vol. 27, no. 6, pp. 1124-1129, 1996.

[20] M. Saito and K. Nakatsugawa, "Increased susceptibility of liver to lipid peroxidation after ingestion of a high fish oil diet," International Journal for Vitamin and Nutrition Research, vol. 64, no. 2, pp. 144-151, 1994.

[21] M. Fujimura, Y. Morita-Fujimura, N. Noshita, T. Sugawara, M. Kawase, and P. H. Chan, "The cytosolic antioxidant copper/ zinc-superoxide dismutase prevents the early release of mitochondrial cytochrome $\mathrm{c}$ in ischemic brain after transient focal cerebral ischemia in mice," The Journal of Neuroscience, vol. 20, no. 8, pp. 2817-2824, 2000.

[22] A. L. Sverdlov, A. Elezaby, F. Qin et al., "Mitochondrial reactive oxygen species mediate cardiac structural, functional, and mitochondrial consequences of diet-induced metabolic heart disease," Journal of the American Heart Association, vol. 5, no. 1, article e002555, 2016.

[23] N. P. Visavadiya, S. P. Patel, J. L. VanRooyen, P. G. Sullivan, and A. G. Rabchevsky, "Cellular and subcellular oxidative stress parameters following severe spinal cord injury," Redox Biology, vol. 8, pp. 59-67, 2016.
[24] T. S. Anthonymuthu, E. M. Kenny, and H. Bayir, "Therapies targeting lipid peroxidation in traumatic brain injury," Brain Research, vol. 1640, pp. 57-76, 2016.

[25] Y. Riahi, G. Cohen, O. Shamni, and S. Sasson, "Signaling and cytotoxic functions of 4-hydroxyalkenals," American Journal of Physiology-Endocrinology and Metabolism, vol. 299, no. 6, pp. E879-E886, 2010.

[26] M. Guichardant, S. Bacot, P. Molière, and M. Lagarde, "Hydroxy-alkenals from the peroxidation of n-3 and n-6 fatty acids and urinary metabolites," Prostaglandins, Leukotrienes and Essential Fatty Acids, vol. 75, no. 3, pp. 179-182, 2006.

[27] M. K. Irmak, E. Fadillioglu, S. Sogut et al., "Effects of caffeic acid phenethyl ester and alpha-tocopherol on reperfusion injury in rat brain," Cell Biochemistry and Function, vol. 21, no. 3, pp. 283289, 2003.

[28] H. K. Heywood and D. A. Lee, "Bioenergetic reprogramming of articular chondrocytes by exposure to exogenous and endogenous reactive oxygen species and its role in the anabolic response to low oxygen," Journal of Tissue Engineering and Regenerative Medicine, 2016.

[29] T. Sugawara, N. Noshita, A. Lewen et al., "Overexpression of copper/zinc superoxide dismutase in transgenic rats protects vulnerable neurons against ischemic damage by blocking the mitochondrial pathway of caspase activation," Journal of Neuroscience, vol. 22, no. 1, pp. 209-217, 2002.

[30] B. Wang, L. Li, J. Fu et al., "Effects of long-chain and mediumchain fatty acids on apoptosis and oxidative stress in human liver cells with steatosis," Journal of Food Science, vol. 81, no. 3, pp. H794-H800, 2016.

[31] E. Sawicka, A. Lisowska, P. Kowal, and A. Długosz, "The role of oxidative stress in bladder cancer," Postępy Higieny i Medycyny Doświadczalnej, vol. 69, pp. 744-752, 2015.

[32] O. A. Ozen, M. Cosar, O. Sahin et al., "The protective effect of fish n-3 fatty acids on cerebral ischemia in rat prefrontal cortex," Neurological Sciences, vol. 29, no. 3, pp. 147-152, 2008.

[33] R. C. S. Seet, C.-Y. J. Lee, B. P. L. Chan et al., "Oxidative damage in ischemic stroke revealed using multiple biomarkers," Stroke, vol. 42, no. 8, pp. 2326-2329, 2011.

[34] H. A. Seifert and K. R. Pennypacker, "Molecular and cellular immune responses to ischemic brain injury," Translational Stroke Research, vol. 5, no. 5, pp. 543-553, 2014.

[35] R. Shirley, E. N. Ord, and L. M. Work, "Oxidative stress and the use of antioxidants in stroke," Antioxidants, vol. 3, no. 3, pp. 472501, 2014.

[36] L. Rebiger, S. Lenzen, and I. Mehmeti, "Susceptibility of brown adipocytes to pro-inflammatory cytokine toxicity and reactive oxygen species," Bioscience Reports, vol. 36, no. 2, Article ID e00306, 2016.

[37] D.-Y. Yang, H.-C. Pan, Y.-J. Yen et al., "Detrimental effects of post-treatment with fatty acids on brain injury in ischemic rats," NeuroToxicology, vol. 28, no. 6, pp. 1220-1229, 2007.

[38] C. Nguemeni, B. Delplanque, C. Rovère et al., "Dietary supplementation of alpha-linolenic acid in an enriched rapeseed oil diet protects from stroke," Pharmacological Research, vol. 61, no. 3, pp. 226-233, 2010.

[39] M. Ueda, T. Inaba, C. Nito, N. Kamiya, and Y. Katayama, "Therapeutic impact of eicosapentaenoic acid on ischemic brain damage following transient focal cerebral ischemia in rats," Brain Research, vol. 1519, pp. 95-104, 2013.

[40] C.-Y. Chang, Y.-H. Kuan, J.-R. Li et al., "Docosahexaenoic acid reduces cellular inflammatory response following permanent 
focal cerebral ischemia in rats," The Journal of Nutritional Biochemistry, vol. 24, no. 12, pp. 2127-2137, 2013.

[41] M. Zhang, S. Wang, L. Mao et al., "Omega-3 fatty acids protect the brain against ischemic injury by activating Nrf2 and upregulating heme oxygenase 1," Journal of Neuroscience, vol. 34, no. 5, pp. 1903-1915, 2014.

[42] H. Esterbauer, R. J. Schaur, and H. Zollner, "Chemistry and biochemistry of 4-hydroxynonenal, malonaldehyde and related aldehydes," Free Radical Biology and Medicine, vol. 11, no. 1, pp. 81-128, 1991.

[43] N. Wakabayashi, A. T. Dinkova-Kostova, W. D. Holtzclaw et al., "Protection against electrophile and oxidant stress by induction of the phase 2 response: fate of cysteines of the Keapl sensor modified by inducers," Proceedings of the National Academy of Sciences of the United States of America, vol. 101, no. 7, pp. $2040-$ 2045, 2004

[44] A. Kobayashi, M.-I. Kang, H. Okawa et al., "Oxidative stress sensor Keapl functions as an adaptor for Cul3-based E3 ligase to regulate proteasomal degradation of Nrf2," Molecular and Cellular Biology, vol. 24, no. 16, pp. 7130-7139, 2004.

[45] B. Xue, Z. Yang, X. Wang, and H. Shi, "Omega-3 polyunsaturated fatty acids antagonize macrophage inflammation via activation of AMPK/SIRT1 pathway," PLoS ONE, vol. 7, no. 10, Article ID e45990, 2012.

[46] Y.-C. Yang, C.-K. Lii, Y.-L. Wei et al., "Docosahexaenoic acid inhibition of inflammation is partially via cross-talk between Nrf2/heme oxygenase 1 and IKK/NF- $\kappa$ B pathways," Journal of Nutritional Biochemistry, vol. 24, no. 1, pp. 204-212, 2013.

[47] L. Zhang, J. Li, J. Ma et al., "The relevance of Nrf2 pathway and autophagy in pancreatic cancer cells upon stimulation of reactive oxygen species," Oxidative Medicine and Cellular Longevity, vol. 2016, Article ID 3897250, 11 pages, 2016.

[48] M. Ploughman, M. W. Austin, L. Glynn, and D. Corbett, "The effects of poststroke aerobic exercise on neuroplasticity: a systematic review of animal and clinical studies," Translational Stroke Research, vol. 6, no. 1, pp. 13-28, 2015.

[49] T. Kawano, J. Anrather, P. Zhou et al., "Prostaglandin E2 EP1 receptors: downstream effectors of COX-2 neurotoxicity," Nature Medicine, vol. 12, no. 2, pp. 225-229, 2006.

[50] J. Zúñiga, M. Cancino, F. Medina et al., "N-3 PUFA supplementation triggers PPAR- $\alpha$ activation and PPAR- $\alpha / N F-\kappa B$ interaction: anti-inflammatory implications in liver ischemiareperfusion injury," PLoS ONE, vol. 6, no. 12, Article ID e28502, 2011.

[51] J.-M. Lee, M. C. Grabb, G. J. Zipfel, and D. W. Choi, "Brain tissue responses to ischemia," Journal of Clinical Investigation, vol. 106, no. 6, pp. 723-731, 2000.

[52] T.-J. Song, H.-J. Cho, Y. Chang et al., "Low plasma proportion of omega 3-polyunsaturated fatty acids predicts poor outcome in acute non-cardiogenic ischemic stroke patients," Journal of Stroke, vol. 17, no. 2, pp. 168-176, 2015.

[53] L. Belayev, L. Khoutorova, K. D. Atkins, and N. G. Bazan, "Robust docosahexaenoic acid-mediated neuroprotection in a rat model of transient, focal cerebral ischemia," Stroke, vol. 40, no. 9, pp. 3121-3126, 2009.

[54] M. D. G. C. de Souza, C. M. S. Conde, C. M. Laflôr, F. L. Sicuro, and E. Bouskela, "N-3 PUFA induce microvascular protective changes during ischemia/reperfusion," Lipids, vol. 50, no. 1, pp. 23-37, 2015.

[55] B. R. Duling, "The preparation and use of the hamster cheek pouch for studies of the microcirculation," Microvascular Research, vol. 5, no. 3, pp. 423-429, 1973.
[56] S. Choi-Kwon, K.-A. Park, H.-J. Lee et al., “Temporal changes in cerebral antioxidant enzyme activities after ischemia and reperfusion in a rat focal brain ischemia model: effect of dietary fish oil," Developmental Brain Research, vol. 152, no. 1, pp. 11-18, 2004.

[57] C. H. Nijboer, M. A. van der Kooij, F. van Bel, F. Ohl, C. J. Heijnen, and A. Kavelaars, "Inhibition of the JNK/AP-1 pathway reduces neuronal death and improves behavioral outcome after neonatal hypoxic-ischemic brain injury," Brain, Behavior, and Immunity, vol. 24, no. 5, pp. 812-821, 2010.

[58] Y. Liu, H. Wang, Y. Zhu, L. Chen, Y. Qu, and Y. Zhu, "The protective effect of nordihydroguaiaretic acid on cerebral ischemia/reperfusion injury is mediated by the JNK pathway," Brain Research, vol. 1445, pp. 73-81, 2012.

[59] Y. Li, D. He, X. Zhang et al., "Protective effect of celastrol in rat cerebral ischemia model: down-regulating p-JNK, p-c-Jun and

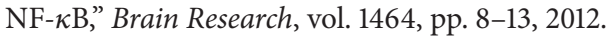

[60] D. Y. Oh, S. Talukdar, E. J. Bae et al., "GPR120 is an omega-3 fatty acid receptor mediating potent anti-inflammatory and insulinsensitizing effects," Cell, vol. 142, no. 5, pp. 687-698, 2010.

[61] K. H. Weylandt, C.-Y. Chiu, B. Gomolka, S. F. Waechter, and B. Wiedenmann, "Omega-3 fatty acids and their lipid mediators: towards an understanding of resolvin and protectin formation. Omega-3 fatty acids and their resolvin/protectin mediators," Prostaglandins and Other Lipid Mediators, vol. 97, no. 3-4, pp. 73-82, 2012.

[62] G. J. E. Rinkel, J. Van Gijn, and E. P. M. Wijdicks, "Subarachnoid hemorrhage without detectable aneurysm: a review of the causes," Stroke, vol. 24, no. 9, pp. 1403-1409, 1993.

[63] J. van Gijn and G. J. E. Rinkel, "Subarachnoid haemorrhage: diagnosis, causes and management," Brain, vol. 124, part 2, pp. 249-278, 2001.

[64] E. J. van Dijk, R. M. M. Hupperts, M. van der Jagt, H. W. C. Bijvoet, and D. Hasan, "Diagnosis of perimesencephalic nonaneurysmal subarachnoid hemorrhage with computed tomography," Journal of Stroke and Cerebrovascular Diseases, vol. 10, no. 6, pp. 247-251, 2001.

[65] J. V. Gijn, K. J. Van Dongen, M. Vermeulen, and A. Hijdra, "Perimesencephalic hemorrhage: a nonaneurysmal and benign form of subarachnoid hemorrhage," Neurology, vol. 35, no. 4, pp. 493-497, 1985.

[66] I. C. van der Schaaf, B. K. Velthuis, A. Gouw, and G. J. E. Rinkel, "Venous drainage in perimesencephalic hemorrhage," Stroke, vol. 35, no. 7, pp. 1614-1618, 2004.

[67] C. P. Marder, V. Narla, J. R. Fink, and K. R. Tozer Fink, "Subarachnoid hemorrhage: beyond aneurysms," American Journal of Roentgenology, vol. 202, no. 1, pp. 25-37, 2014.

[68] N. Etminan, "Aneurysmal subarachnoid hemorrhage-status quo and perspective," Translational Stroke Research, vol. 6, no. 3, pp. 167-170, 2015.

[69] M. Selim and K. N. Sheth, "Perihematoma edema: a potential translational target in intracerebral hemorrhage?" Translational Stroke Research, vol. 6, no. 2, pp. 104-106, 2015.

[70] H. H. Dietrich and R. G. Dacey Jr., "Molecular keys to the problems of cerebral vasospasm," Neurosurgery, vol. 46, no. 3, pp. 517-530, 2000.

[71] X.-Y. Xiong and Q.-W. Yang, "Rethinking the roles of inflammation in the intracerebral hemorrhage," Translational Stroke Research, vol. 6, no. 5, pp. 339-341, 2015.

[72] J. J. Provencio and N. Vora, "Subarachnoid hemorrhage and inflammation: bench to bedside and back," Seminars in Neurology, vol. 25, no. 4, pp. 435-444, 2005. 
[73] B. Lucke-Wold, A. Logsdon, B. Manoranjan et al., "Aneurysmal subarachnoid hemorrhage and neuroinflammation: a comprehensive review," International Journal of Molecular Sciences, vol. 17, no. 4, article 497, 2016.

[74] M. Sato, E. Tani, H. Fujikawa, and K. Kaibuchi, "Involvement of Rho-kinase-mediated phosphorylation of myosin light chain in enhancement of cerebral vasospasm," Circulation Research, vol. 87, no. 3, pp. 195-200, 2000.

[75] S. Yoon, J. D. Sherman, M. Zuccarello, and R. M. Rapoport, "Vasospasm following subarachnoid hemorrhage: evidence against functional upregulation of Rho kinase constrictor pathway," Neurological Research, vol. 24, no. 4, pp. 392-394, 2002.

[76] T. Koji, Y. Nishikawa, M. Doi, K. Sakaki, and A. Ogawa, "Augmenting mechanism of contractile response to the stimulation of thromboxane A2-receptor in the middle cerebral artery of bovine," The Japanese Society on Surgery for Cerebral Stroke, vol. 30, pp. 41-45, 2002.

[77] S. Shirao, S. Kashiwagi, M. Sato et al., "Sphingosylphosphorylcholine is a novel messenger for rho-kinase-mediated $\mathrm{Ca}^{2+}$ sensitization in the bovine cerebral artery: unimportant role for protein kinase C," Circulation Research, vol. 91, no. 2, pp. 112-119, 2002.

[78] F. Nakao, S. Kobayashi, K. Mogami et al., "Involvement of Src family protein tyrosine kinases in $\mathrm{Ca}^{2+}$ sensitization of coronary artery contraction mediated by a sphingosylphosphorylcholine-Rho-kinase pathway," Circulation Research, vol. 91, no. 10, pp. 953-960, 2002.

[79] J. G. Pilitsis, W. M. Coplin, M. H. O’Regan et al., "Free fatty acids in human cerebrospinal fluid following subarachnoid hemorrhage and their potential role in vasospasm: a preliminary observation," Journal of Neurosurgery, vol. 97, no. 2, pp. 272-279, 2002.

[80] A. Hirai, T. Terano, T. Hamazaki et al., "The effects of the oral administration of fish oil concentrate on the release and the metabolism of $\left[{ }^{14} \mathrm{C}\right]$ arachidonic acid and $\left[{ }^{14} \mathrm{C}\right]$ eicosapentaenoic acid by human platelets," Thrombosis Research, vol. 28, no. 3, pp. 285-298, 1982.

[81] H. Yoneda, S. Shirao, T. Kurokawa, H. Fujisawa, S. Kato, and M. Suzuki, "Does eicosapentaenoic acid (EPA) inhibit cerebral vasospasm in patients after aneurysmal subarachnoid hemorrhage?" Acta Neurologica Scandinavica, vol. 118, no. 1, pp. 54-59, 2008.

[82] H. Yoneda, S. Shirao, J. Nakagawara, K. Ogasawara, T. Tominaga, and M. Suzuki, "A prospective, multicenter, randomized study of the efficacy of eicosapentaenoic acid for cerebral vasospasm: the EVAS study," World Neurosurgery, vol. 81, no. 2, pp. 309-315, 2014.

[83] N. Badjatia, D. Seres, A. Carpenter et al., "Free fatty acids and delayed cerebral ischemia after subarachnoid hemorrhage," Stroke, vol. 43, no. 3, pp. 691-696, 2012.

[84] H. S. Pedersen, G. Mulvad, K. N. Seidelin, G. T. Malcom, and D. A. Boudreau, "N-3 fatty acids as a risk factor for haemorrhagic stroke," The Lancet, vol. 353, no. 9155, pp. 812-813, 1999.

[85] Y. Park, S. Nam, H.-J. Yi, H.-J. Hong, and M. Lee, "Dietary n-3 polyunsaturated fatty acids increase oxidative stress in rats with intracerebral hemorrhagic stroke," Nutrition Research, vol. 29, no. 11, pp. 812-818, 2009.

[86] J. Clarke, G. Herzberg, J. Peeling, R. Buist, and D. Corbett, "Dietary supplementation of omega-3 polyunsaturated fatty acids worsens forelimb motor function after intracerebral hemorrhage in rats," Experimental Neurology, vol. 191, no. 1, pp. 119127, 2005. 


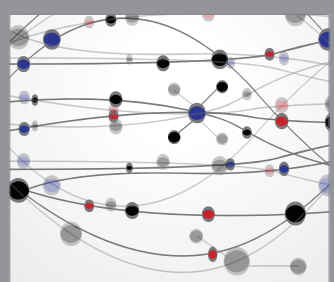

The Scientific World Journal
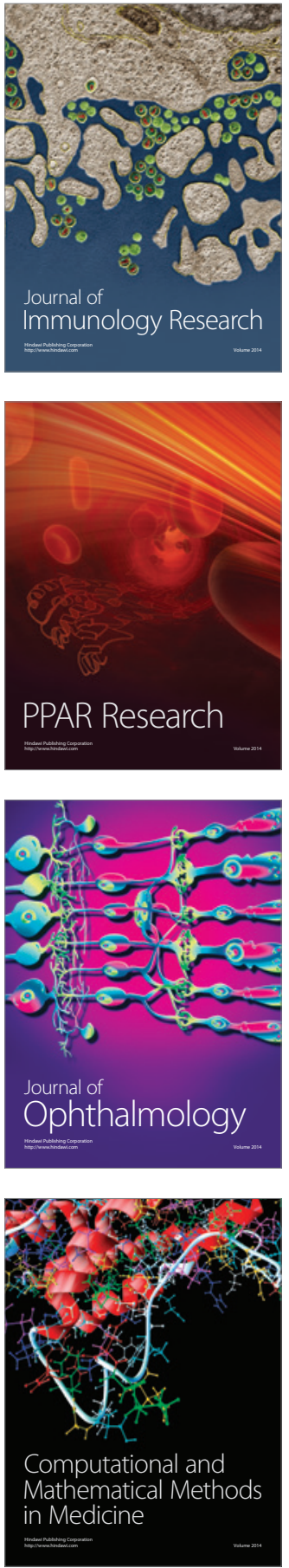

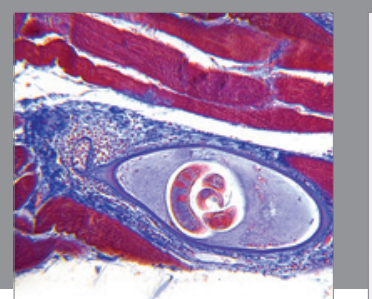

Gastroenterology Research and Practice

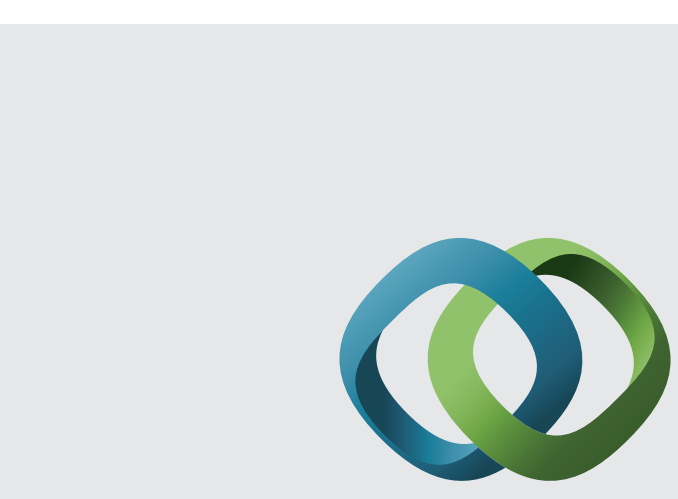

\section{Hindawi}

Submit your manuscripts at

http://www.hindawi.com
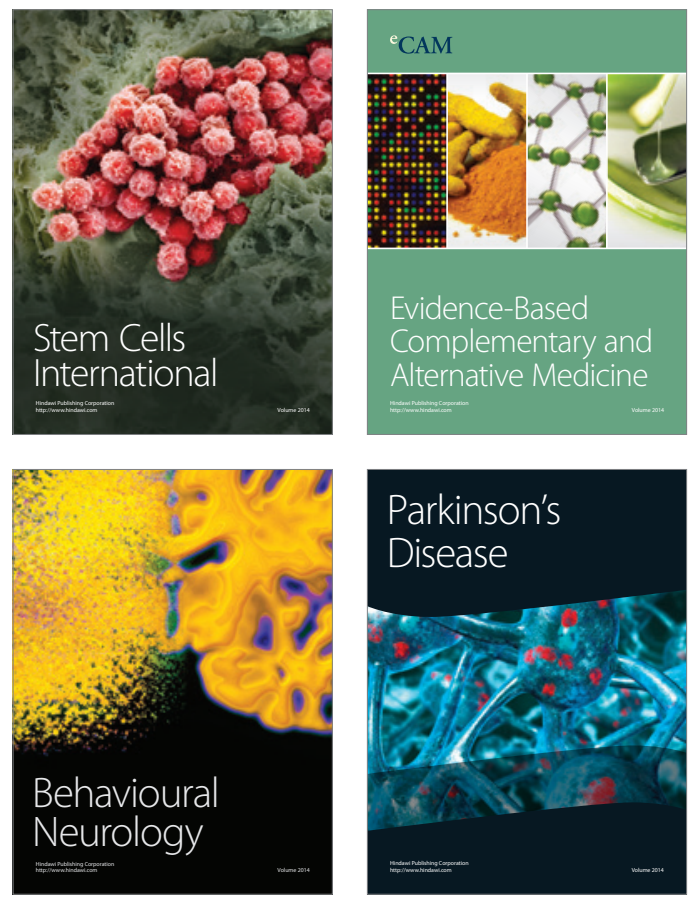
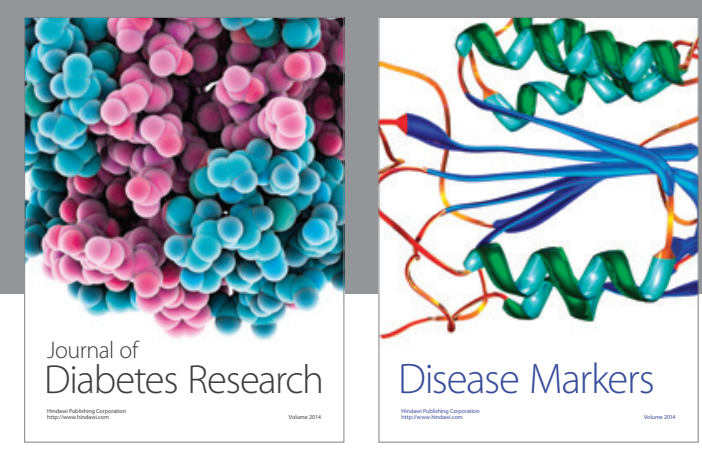

Disease Markers
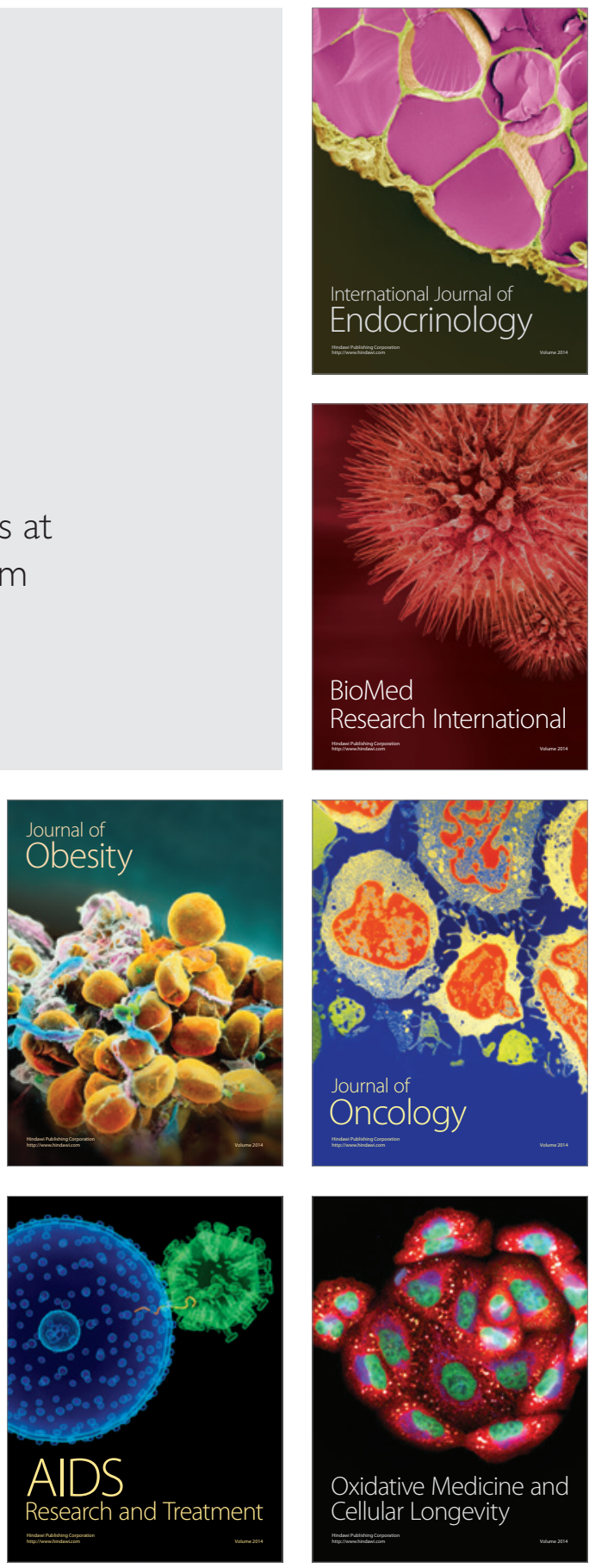